\title{
The dynamical temperature and the standard map
}

\author{
I. I. Shevchenko* \\ Pulkovo Observatory of the Russian Academy of Sciences \\ Pulkovskoje ave. 65/1, St.Petersburg 196140, Russia
}

\begin{abstract}
Numerical experiments with the standard map at high values of the stochasticity parameter reveal the existence of simple analytical relations connecting the volume and the dynamical temperature of the chaotic component of the phase space.
\end{abstract}

Key words: Hamiltonian dynamics, chaotic dynamics, standard map, dynamical temperature.

\section{Introduction}

The standard map is an important object in studies in nonlinear dynamics, mainly because it is often used to describe the local behavior of other more complicated symplectic maps [1, 2, 3. What is more, it serves as an important independent mechanical and physical paradigm [4, 5]. General properties of the standard map, often in interrelation with those of the separatrix map, Fermi map and other fundamental maps, were considered and studied in detail in [1]-[13], and many other works.

The standard map is given by the equations

*E-mail: iis@gao.spb.ru 


$$
\begin{aligned}
y_{i+1} & =y_{i}+\frac{K}{2 \pi} \sin \left(2 \pi x_{i}\right) \quad(\bmod 1), \\
x_{i+1} & =x_{i}+y_{i+1}(\bmod 1)
\end{aligned}
$$

where $K$ is the so-called stochasticity parameter [1, 2,

In Ref. [10] we studied three major characteristics of the chaotic dynamics of the standard map, namely, the measure $\mu$ of the main connected chaotic domain (MCCD), the maximum Lyapunov exponent $L$ of the motion in this domain, and the dynamical entropy $h=\mu L$, as functions of the stochasticity parameter $K$. The perturbations of the domain due to the birth and disintegration of islands of stability, upon variations of $K$, were considered in particular. By means of extensive numerical experiments, we showed that these perturbations are isentropic, at least approximately: the dynamical entropy follows the smooth dependence $h(K)=\ln \frac{K}{2}+\frac{1}{K^{2}}$, and does not fluctuate about it with changing the stochasticity parameter, while local jumps in $\mu$ and $L$ due to the birth and disintegration of regular islands are significant.

On the other hand, recently Baldovin [7] and Baldovin et al. [8] introduced the notion of the "dynamical temperature" for the standard map, defining it as the variance of the momentum:

$$
T \equiv\left\langle(y-\langle y\rangle)^{2}\right\rangle-\frac{1}{12}=\left\langle y^{2}\right\rangle-\langle y\rangle^{2}-\frac{1}{12},
$$

where the angle brackets signify ensemble average; the constant addend is the normalizing shift, introduced for convenience. This choice of normalization gives $T=0$ for the case of the uniform ensemble [7, 8]. Henceforth we use different normalization:

$$
T \equiv 12\left\langle(y-\langle y\rangle)^{2}\right\rangle=12\left(\left\langle y^{2}\right\rangle-\langle y\rangle^{2}\right) .
$$

This gives $T=1$ for the case of the uniform ensemble. This normalization is used in calculating $T$ in what follows. In practice, the measurement of $T$ is performed by averaging over an iterated trajectory in the chaotic domain, i.e., we assume that the ensemble average is equal to the time average. This is justified in the considered situation, when the regular islands are small and the motion is almost completely ergodic on the phase plane. The relation between ensemble and time averages for low-dimensional symplectic maps in the opposite case of extended borders between chaotic and regular components of the phase space were considered and analyzed in Ref. [7]. 
In this paper, we investigate the behavior of two state variables of the MCCD of the phase space of the standard map: namely, the volume $\mu$ and the dynamical temperature $T$, both as functions of the stochasticity parameter $K$. At moderate values of $K$ (at approximately $K<4$ ), the non-monotonic (spike) variations in $\mu(K)$ and $T(K)$ are conditioned by the process of absorption of minor chaotic domains by the MCCD, while $K$ increases; at larger values of $K$ (at approximately $K>4$ ), they are conditioned by the process of birth and disintegration of stability islands. In other words, the perturbations of the chaotic domain at high values of $K$ are due to the birth and disintegration of islands of stability, upon small variations of $K$. By means of extensive numerical experiments, we show that the variations of $\mu$ and $T$ due to this process obey simple analytical relations.

\section{Numerical experiments}

The traditional "one trajectory method" (OTM) [1, 2, 14, 10] has been used for calculation of $\mu$. This method consists in computing the number of cells explored by a single trajectory on a grid exposed on the phase plane. A strict but computationally much more expensive approach for measuring $\mu$ consists in calculating the values of the coarse-grained area of the chaotic component for a set of various resolutions of the grid, in order to find the asymptotic value of $\mu$ at the infinitely fine resolution (see [12]). In some cases the "LCE segregation method" (LCESM) [1, 2, 14, 10] has been employed for verification of the obtained values of $\mu$. In this method, a numeric criterion is used for separation of the regular and chaotic trajectories. The criterion is provided by an analysis of the differential distribution of the values of the finite-time Lyapunov characteristic exponents (LCEs) computed on a set of trajectories with the starting values randomly generated on the phase plane or specified on a regular grid on the phase plane. In the distribution, the peak corresponding to the regular trajectories is movable: if one increases the LCE computation time, the peak moves to the left on the abscissa axis, because the computed LCEs for the regular trajectories tend to zero; while all the peaks corresponding to the chaotic domains stay immovable, on condition that the computation time is long enough. By increasing the computation time one can make the movable and immovable peaks completely distinct, and in this way determine the "finite-time LCE" value separating the regular and chaotic trajectories for the given computation time. The OTM and LCESM 
were both proposed and used by Chirikov [1, 2] in computations of $\mu$ for the standard map. Analogous methods were used in Ref. [14 in computations of the chaotic domain measure in the Hénon-Heiles problem, and in Ref. [10] for computations of $\mu$ for the standard map. A detailed description of the currently used versions of the methods is given in Ref. [10].

In Figs. 1-4, we present the obtained numerical data on the measure $\mu$ and the dynamical temperature $T$ of the MCCD, and their interrelations. Fig. 1 shows $\mu(K)$ and $T(K)$ for a perturbation of the MCCD due to the birth and disintegration of the regular islands corresponding to a 2-periodic solution (at $K \in(11.9,12.6)$ ) and to an accelerator mode (at $K \in(12.6,13.3)$ ). An accelerator mode represents a 1 -periodic solution on the torus $x, y \in$ $[0,1] \times[0,1]$. Theoretical ranges in $K$ for the existence of both types of solutions are given in [2] the narrow "windows" of their existence follow with the period of $2 \pi$ in $K$, and with increasing $K$ they become more and more narrow. In Fig. 2, the same plots are constructed for a perturbation conditioned by the birth and decay of the islands due to a 4 -periodic solution at $K \approx 9.21 \div 9.45$.

The OTM has been used for calculating $\mu$; the grid is $5000 \times 5000$ pixels on the square $x, y \in[0,1] \times[0,1]$. The map has been iterated $n_{i t}=10^{9}$ times at each value of $K$. The step in $K$ is equal to 0.005 (Fig. 1) and 0.001 (Fig. 2). Each value of $T$ has been computed simultaneously with the value of $\mu$ for the same trajectory.

No error bars are shown in the Figs. 1 and 2, since the typical errors appear to be rather low. One can estimate them in the following way. Consider first the calculation of $\mu$. The main uncertainty in the measured $\mu$ value, at sufficiently long iteration times $n_{i t}$, is conditioned by the resolution of the grid imposed on the phase plane. We designate the number of pixels at each side of the square $x, y \in[0,1] \times[0,1]$ by $l$; so, $l=5000$. Consider the model case of a round islet (the choice of geometries is not principal for our estimate by the order of magnitude). The relative uncertainty $\varepsilon$ in the measured area of the disk is given approximately by one half of the ratio of the area of the chain of the pixels at the disk border and the disk area $S$. It is straightforward to show that $\varepsilon=l^{-1}(\pi / S)^{1 / 2}$. This is the relative error by which the island area can be overestimated by the OTM. For the accelerator modes the maximum area of one island scales as $0.19 K^{-2}$ [4]; so, one has $\varepsilon \approx 4 K l^{-1}$. For the accelerator mode in Fig. 1 this formula gives $\varepsilon \approx 0.010$. This is the relative uncertainty in the measure $\mu_{\text {reg }}$ of the regular component of the phase space. Hence the absolute uncertainty in $\mu$ (in the ordinate in 
Fig. 1) is equal to $2 \cdot 10^{-5}$; i.e., the error is smaller than the size of the data point symbols in the Figure. Practically the same error values are derived in the case of the plot in Fig. 2, i.e., in the case of the 4-periodic solution.

In what concerns the errors in calculation of $T$, they seem to be controlled only by the computation time $n_{i t}$. If one decreases this time, the "noise" appears in the plot: instead of the smooth long-range (in comparison with the step in $K$ ) variations, $T$ varies irregularly at each step in $K$; the amplitude of these irregular one-step variations gives the typical error in the estimation of $T$. From the fact of the absence of such variations in Figs. 1 and 2, one concludes that the errors are typically smaller than at least one-half of the distance in $\mu$ between each two consecutive points on the plots, i.e., they are insignificant in the given case of $n_{i t}=10^{9}$.

\section{Analytical relations between volume and dynamical temperature}

The local minima in $\mu(K)$ seen in Figs. 1 and 2 clearly correspond to local extrema in $T(K)$. The observed amplitudes of the volume and temperature variations are apparently commensurable, namely, their ratios represent some integer or half-integer numbers. This suggests the existence of the power law relations with rational exponents. We verify this hypothesis by constructing the " $\mu-T$ " graphs in Figs. 3 and 4. All the points present in Figs. 1 and 2 are depicted in Figs. 3 and 4; in the latter Figures, each pair of neighbors in $K$ are connected by straight segments.

For all $K>5$ the islands are small, i.e., $\mu$ is close to 1 ; this is discussed in detail at the end of this Section. In this context, it clearly follows from Figs. 3 and 4 that the relation

$$
T=1-\gamma(1-\mu)
$$

takes place with three different values of the $\gamma$ constant. Namely, $\gamma=-1$ in the case of the 2-periodic solution, $\gamma=2$ for the accelerator mode, and $\gamma=1 / 2$ for the 4 -periodic solution.

Among 320 data points present in Fig. 3 there are 10 (i.e., $\approx 3 \%$ ) that notably (by more than 0.0005) deviate from the dotted lines given by Eq. (41) with $\gamma=-1$ and 2 ; among 350 data points in Fig. 4 there are 8 (i.e., $\approx 2 \%$ ) that notably (by more than 0.0002) deviate from the dotted line given by 
Eq. (4) with $\gamma=1 / 2$. In other words, the percentage of notable deviations is rather low. The deviating points apparently correspond to those values of $K$ at which secondary resonance chains separate from the outer borders of the islands; these phenomena manifest themselves in the secondary peaks in the curves $\mu(K)$ and $T(K)$. In such situations the measuring of both $\mu$ and $T$ are subject to additional uncertainties: the thin chaotic layers between the islands and the surrounding resonance chains can be unresolved in the adopted grid of pixels, and, besides, the enhanced sporadic sticking of the chaotic trajectories to the islands may lead to increasing the contribution of the "temperature on the islands" (this notion is considered below) in the total measured temperature value.

Apart from these casual deviations, the sticking phenomenon does not influence the temperature measurements. (This is contrary to the case of the diffusion coefficient, which, as it is well known, diverges in the presence of the accelerator modes, see [2, 4].) If a trajectory constantly sticks to an accelerator mode island, the temperature tends to the "temperature on the island" value, which is finite, because the temperature (2) and (3) is defined in the factored phase space: $y$ is taken modulo 1 .

If one puts Eq. (41) in the form $\frac{\Delta T}{T}=\gamma \frac{\Delta \mu}{\mu}$ and integrates, one has

$$
T=\mu^{\gamma} .
$$

Eqs. (44) and (5) are practically equivalent for any application. At $K=9.24$, the MCCD area $\mu \approx 0.995$; and the values of $T$ given by Eqs. (41) and (5) at this value of $\mu$ differ by only $2.5 \cdot 10^{-5}$. For smaller islands, emerging at greater values of $K$, the difference is smaller.

We have considered the behavior of the standard map at two definite intervals of $K$. Now let us look at the problem in a global perspective. In Fig. 5, the dependences $\mu(K)$ and $T(K)$ are presented in a broad range: $K \in[4,30]$. The values of $\mu(K)$ and $T(K)$ are calculated as previously, except that a lower grid resolution $(2000 \times 2000$ pixels $)$ and shorter trajectories $\left(n_{i t}=10^{8}\right)$ are used. The step in $K$ is equal to 0.01 . The observed $\mu(K)$ dependence (Fig. 5) at large values of $K$, i.e., at $K$ approximately greater than 6 , represents the basic line $\mu=1$ interrupted by periodic sequences of sharp narrow minima whose depth diminishes with $K$. The most prominent of these sequences correspond to the 2-periodic solutions and accelerator modes (at $K_{m} \approx 2 \pi m ; m=1,2, \ldots$ ) and the 4-periodic solutions (at $\left.K_{m} \approx 2 \pi\left(m+\frac{1}{2}\right) ; m=1,2, \ldots\right)$. The less pronounced sequences of 
minima correspond to periodic solutions of higher order. The observed minimum values of $\mu$ for these three basic cases of periodic solutions agree with the semi-analytical scaling $\mu_{r e g}=0.38 K^{-2}$ derived by Chirikov [4] for the maximum area of the accelerator mode islands. The difference is within $(1 \div 2) \cdot 10^{-4}$. This agreement verifies the good accuracy of our measurements of $\mu$. Note that recently Giorgilli and Lazutkin [6] rigorously derived the inversely quadratic law of decay with $K$ of the area of the main regular islands, i.e., the islands emerging at $K \approx \pi n(n=2,3, \ldots)$.

The variations with $K$ in the standard map behavior due to the 2-periodic solutions and accelerator modes repeat themselves at $m=2,3, \ldots$, only the amplitude of the variations diminish. The same is true for the 4-periodic solutions. So, it is natural to expect that the laws (4) and (5) are universal for all $m$. We have performed recomputation of our plots taking $K \in[18.4,19.3]$ (a 2-periodic solution and an accelerator mode, $m=3$ ) and $K \in[15.58,15.67]$ (a 4-periodic solution, $m=2$ ). As expected, the values of $\gamma$ have been found to reproduce the cases analyzed above.

\section{Theoretical explanation}

Finally, we consider a tentative explanation for the observed relations (4) and (51). In Ref. [10], we showed that the dynamical entropy $h=\mu L$, where $L$ is the maximum Lyapunov exponent of the motion in the MCCD, follows the smooth dependence $h(K)=\ln \frac{K}{2}+\frac{1}{K^{2}}$, and does not fluctuate about it with changing $K$, while local jumps in $\mu$ and $L$ due to the birth and disintegration of regular islands are significant. In other words, the $h(K)$ function normalized by the "long-term" trend $\ln \frac{K}{2}+\frac{1}{K^{2}}$ is constant and thus represents an invariant with respect to $K$. Could a similar invariant be found in the case of the dynamical temperature? Note that the dynamical entropy $h=\mu L$ is nothing but the maximum Lyapunov exponent averaged over the whole phase space, because the maximum Lyapunov exponent is zero on the islands. Since the dynamical temperature is, generally speaking, non-zero on the islands, an analogous temperature invariant, which is the dynamical temperature averaged over the whole phase space including the chaotic sea and the islands, would have the form $\Theta=\mu_{\text {sea }} T_{\text {sea }}+\mu_{\text {isles }} T_{\text {isles }}=1$ in our normalization of $T$. For the sake of clarity we designate $\mu_{\text {sea }} \equiv \mu, T_{\text {sea }} \equiv T$; $\mu_{\text {isles }}$, equal to $1-\mu$, is the measure of the islands; $T_{i s l e s}$ is the temperature on the islands, i.e., the quantity (3) calculated for the initial data on the 
islands. From this equality one has

$$
T=\frac{1-\mu_{\text {isles }} T_{i s l e s}}{1-\mu_{\text {isles }}}=1+\mu_{\text {isles }}\left(1-T_{\text {isles }}\right)+O\left(\mu_{\text {isles }}^{2}\right) .
$$

Comparing Eqs. (44) and (6) with the precision of $O\left(\mu_{i s l e s}^{2}\right)$, we find

$$
\gamma \approx T_{\text {isles }}-1
$$

The quantity $T_{\text {isles }}$ is determined by the $y$ location of the islands in the phase space. The data on the location of the islands is available in [2] (for the 2-periodic solutions and accelerator modes) and in [1] (for the 4-periodic solutions). The temperature is straightforwardly calculated by Eq. (3), and it has the values: $T_{i s l e s} \approx 0,3$, and $3 / 2$ for the 2 -periodic solutions, accelerator modes, and 4-periodic solutions, respectively; i.e., $\gamma \approx-1,2$, and $1 / 2$, respectively, in accord with our analysis of the plots in Figs. 3 and 4.

\section{Conclusions}

Let us summarize the main results. The measure $\mu$ of the main connected chaotic domain (MCCD) of the standard map and its dynamical temperature $T$ have been studied as functions of the stochasticity parameter $K$ by means of extensive numerical experiments. The process of the birth and disintegration (upon small variations of $K$ ) of the islands of stability inside the chaotic domain result in local variations (spikes) in $\mu(K)$ and $T(K)$. It has been shown that the variations of $\mu$ and $T$ are connected by certain dependences (44). Since the island areas are small, these dependences are equivalent to power laws (5): $T=\mu^{\gamma}$, with $\gamma=-1$ and 2 for the 2-periodic solutions and accelerator modes respectively and $\gamma=1 / 2$ for the 4-periodic solutions. It is interesting to note that the value of $\gamma$ for the 4-periodic solutions is the arithmetic mean of the values for the 2-periodic solutions and accelerator modes.

If the newly found relations are confirmed analytically in a rigorous way, they would provide an efficient instrument for estimating the measure of chaotic domains by means of computing the dynamical temperature. Such a method would be more efficient than the OTM or LCESM, since any direct measurement of $\mu$ is a tedious and time-consuming task, subject to a number of uncertainties. 
The author is thankful to anonymous referees for advice and remarks which led to significant improvement of the manuscript. This work was supported by the Russian Foundation for Basic Research (project \# 0502-17555) and by the Programme of Fundamental Research of the Russian Academy of Sciences "Fundamental Problems in Nonlinear Dynamics". The computations were partially carried out at the St. Petersburg Branch of the Joint Supercomputer Centre of the Russian Academy of Sciences.

\section{References}

[1] B. V. Chirikov, Interaction of Nonlinear Resonances, Novosibirsk State University, Novosibirsk, 1978 [in Russian].

[2] B. V. Chirikov, Phys. Rep. 52 (1979) 263.

[3] A. J. Lichtenberg, M. A. Lieberman, Regular and Chaotic Dynamics, Springer-Verlag, New York, 1992.

[4] B. V. Chirikov, Zh. Eksp. Teor. Fiz. 110 (1996) 1174 [JETP 83 (1996) 646].

[5] J. D. Meiss, Phys. Rep. 64 (1992) 795.

[6] A. Giorgilli, V. F. Lazutkin, Phys. Lett. A 272 (2000) 359.

[7] F. Baldovin, Physica A 342 (2004) 119.

[8] F. Baldovin, E. Brigatti, C. Tsallis, Phys. Lett. A 320 (2004) 254.

[9] I. I. Shevchenko, Pis'ma Zh. Eksp. Teor. Fiz. 79 (2004) 651 [JETP Letters 79 (2004) 523].

[10] I. I. Shevchenko, Phys. Letters A 333 (2004) 408.

[11] D. V. Treshchev, An Introduction to the Perturbation Theory of Hamiltonian Systems, FAZIS, Moscow, 1998 [in Russian].

[12] D. K. Umberger, J. D. Farmer, Phys. Rev. Letters 55 (1985) 661.

[13] V. V. Vecheslavov, B. V. Chirikov, Zh. Eksp. Teor. Fiz. 114 (1998) 1516 [in Russian]. 
[14] I. I. Shevchenko, A. V. Melnikov, Pis'ma Zh. Eksp. Teor. Fiz. 77 (2003) 772 [JETP Letters 77 (2003) 642]. 


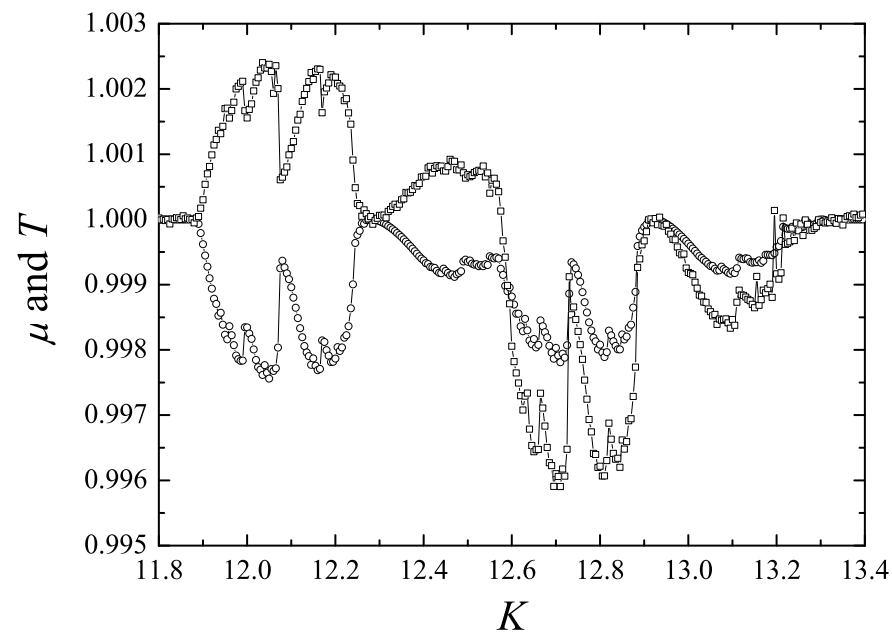

Figure 1: The volume $\mu$ (circles) and the dynamical temperature $T$ (squares) of the MCCD, in function of $K$, for a perturbation due to a 2-periodic solution and an accelerator mode.

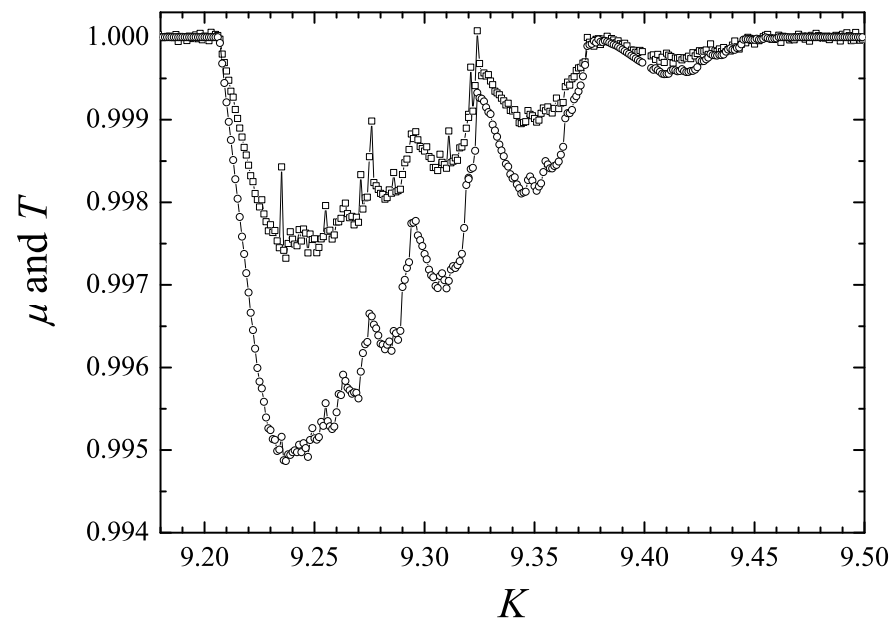

Figure 2: The same for a perturbation due to a 4-periodic solution. 


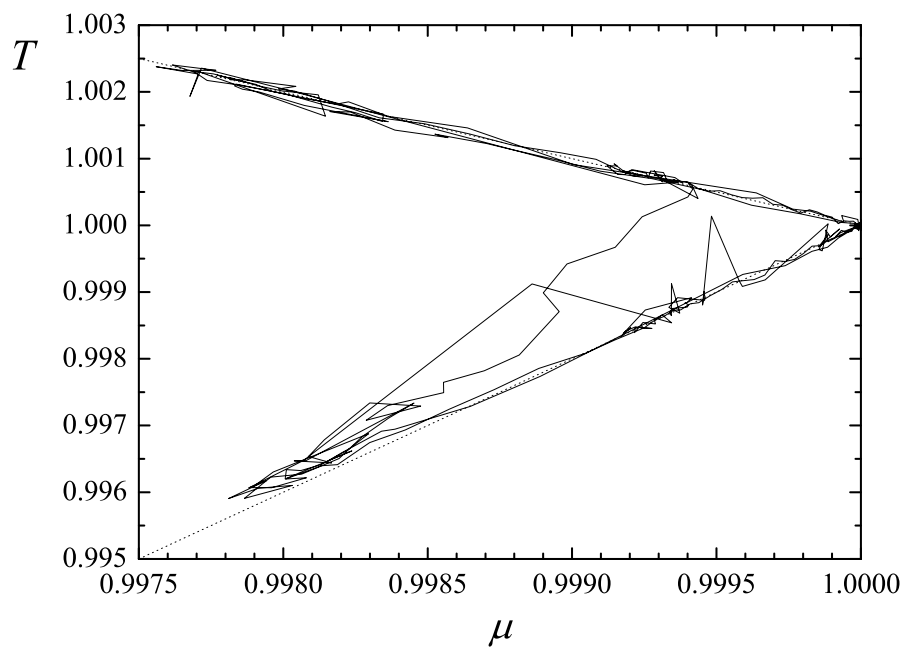

Figure 3: The " $\mu-T$ " relation (solid polygonal line) for the perturbation shown in Fig. 1. Relations (5) with $\gamma=-1$ and 2 are given by the dotted straight lines. 


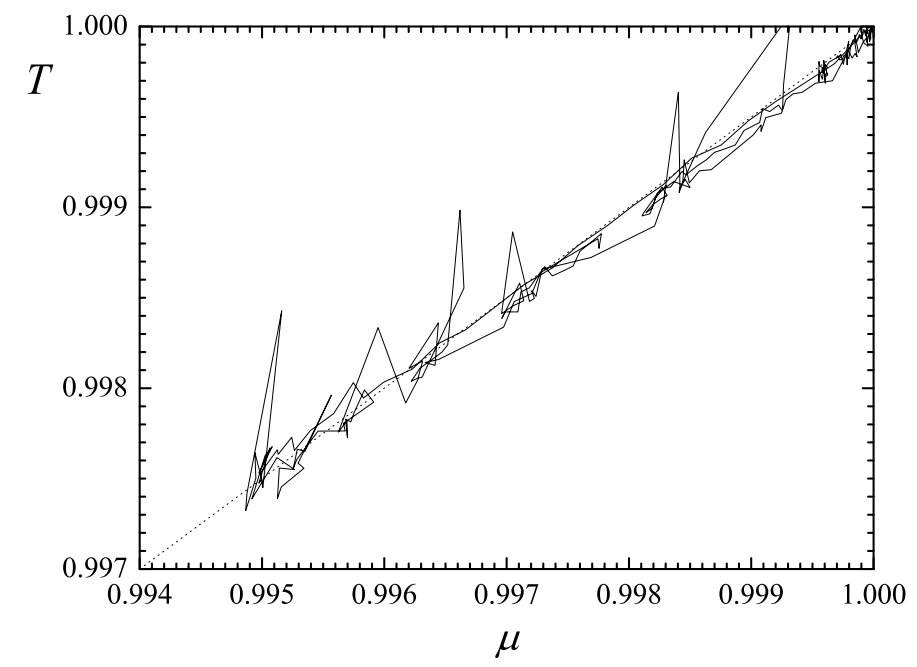

Figure 4: The " $\mu-T$ " relation (solid polygonal line) for the perturbation shown in Fig. 2. Relation (5) with $\gamma=1 / 2$ is given by the dotted straight line. 


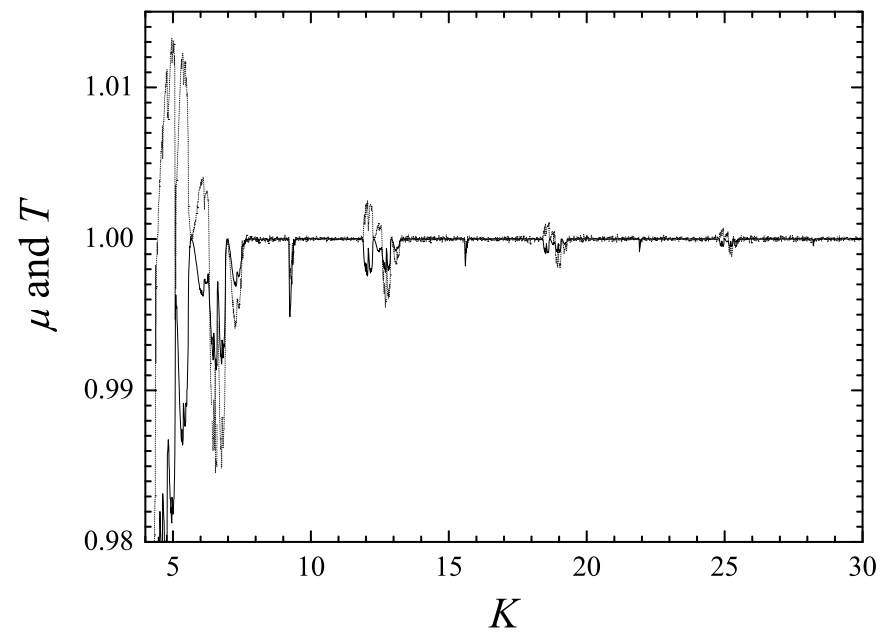

Figure 5: The $\mu(K)$ and $T(K)$ dependences (dark solid and pale dotted curves, respectively) in a broad range of $K$. Note the periodic appearance of patterns of similar structure. 